\title{
Resonant (Longitudinal and Transverse) Electron Polarimetry
}

\author{
R. Talman, ${ }^{*, a}$ B. Roberts, ${ }^{b}$ J. Grames, ${ }^{c}$ A. Hofler, ${ }^{c}$ R. Kazimi, ${ }^{c}$ M. Poelker ${ }^{c}$ \\ and R. Suleiman ${ }^{c}$ \\ ${ }^{a}$ LEPP, ${ }^{c}$ Cornell University \\ ${ }^{b}$ University of New Mexico \\ ${ }^{c}$ Thomas Jefferson National Laboratory \\ E-mail: richard.talman@cornell.edu
}

\begin{abstract}
Experiments are proposed to measure (first longitudinal, then, later, using Stern-Gerlach (SG) deflection, transverse) polarization of an electron beam by measuring the excitation induced in a resonant cavity. In each case there are two major difficulties. The polarimetry signals are very weak, making them hard to detect in absolute terms and, even more serious, is the smallness of the SG signals relative to imperfection-induced, direct excitation of the resonant detector by the beam charge. With ideal resonator construction and positioning, this background would vanish. But, because the electron charge is so large relative to its magnetic moment, special beam preparation and polarization modulation are required to suppress this background. Signal levels and foreground to background ratios are calculated.
\end{abstract}

2017 International Workshop on Polarized Sources, Targets \& Polarimetry Daejeon 34051, Republic of Korea,

Oct 16-20, 2017

\footnotetext{
* Speaker.
} 


\section{CEBAF polarized beam preparation}

Dual CEBAF electron sources (at the Thomas Jefferson National Laboratory) produce oppositely polarized A and B beams having bunch separation $4 \mathrm{~ns}$. Interleaved, the resulting A \& B beam has bunch separation $2 \mathrm{~ns}$. The effect of this beam preparation is to produce a bunch charge repetition frequency of $0.5 \mathrm{GHz}$, different from the bunch polarization frequency of $0.25 \mathrm{GHz}$. With frequency domain spectral filtering this frequency separation will greatly enhance the foreground/background selectivity.

Because linac bunches are short there is substantial resonator response at numerous strong low order harmonics of the $0.25 \mathrm{GHz}$ bunch polarization frequency. The proposed polarimetry responses (referred to here also as Stern-Gerlach (SG) responses), are centered at odd harmonics, $f_{r}=0.25,0.75,1.25, \ldots \mathrm{GHz}$. The absence of beam-induced detector response at these odd harmonics greatly improves the rejection of spurious "background" caused by bunch charge combined with apparatus imperfection and misalignment.

For further background rejection the polarization amplitudes are modulated at a low, $\mathrm{kHz}$, frequency, which shifts the SG response to sidebands of the central SG frequencies.

Exactly the same beam preparation being discussed in this section will be needed both for longitudinal and transverse SG- polarimetry, which are discussed in subsequent sections.

Current and polarization time domain beam amplitudes are plotted on the left in the Figure 1, and their frequency domain signals are plotted on the right, confirming the previous qualitative discussion.

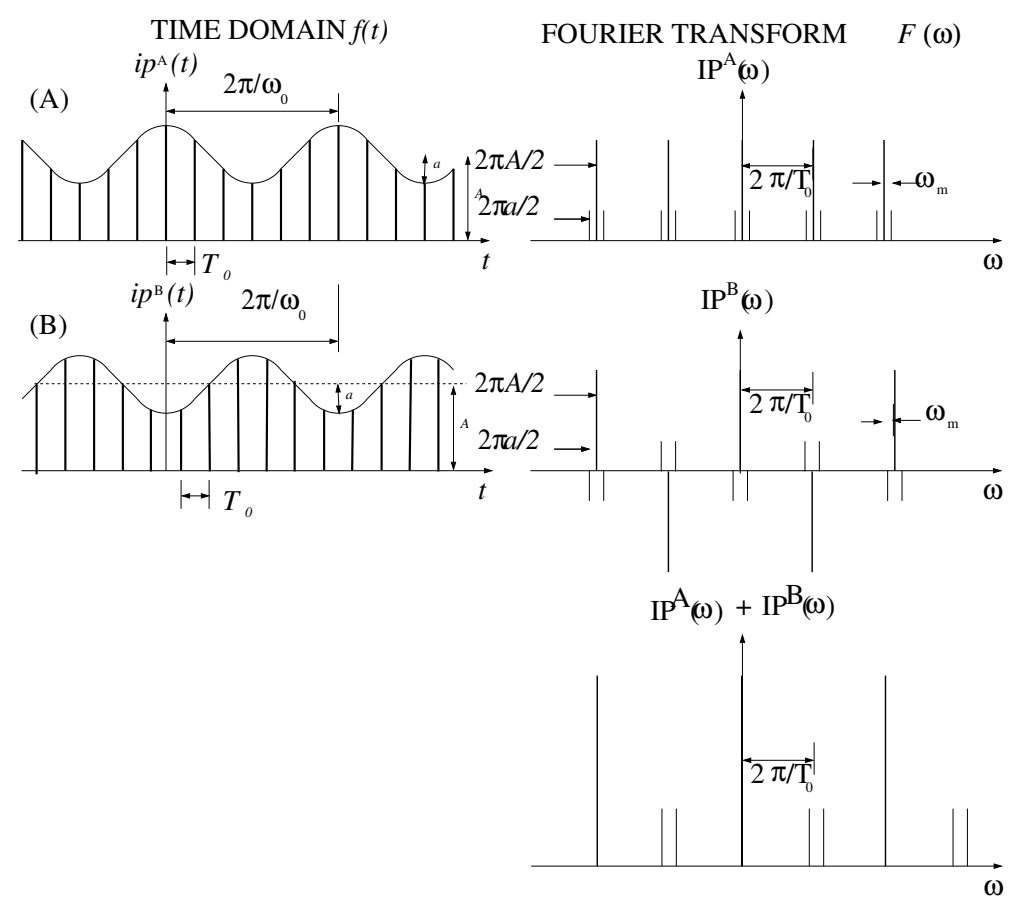

Figure 1: Current and polarization time domain beam amplitudes are plotted on the left, and their frequency domain signals are plotted on the right. Charge sensitive resonator responses appear at harmonics of $0.5 \mathrm{GHz}$, polarization sensitive responses appear at (modulation side-bands) of odd harmonics of $0.25 \mathrm{GHz}$ 
The fundamental impediment to resonant electron polarimetry comes from the smallness of the ratio of magnetic moment divided by charge,

$$
\frac{\mu_{B} / c}{e}=1.930796 \times 10^{-13} \mathrm{~m} .
$$

(Except for a tiny anomalous magnetic moment correction and sign, the electron magnetic moment is equal to the Bohr magneton $\mu_{B}$.) This ratio has the dimension of length because the SternGerlach force due to magnetic field acting on $\mu_{B}$, is proportional to the gradient of the magnetic field.

To the extent that it is "natural" for the magnitudes of $E$ and $c B$ to be comparable, SternGerlach forces are weaker than electromagnetic forces by ratio (1.1). This adverse ratio needs to be overcome (by beam, apparatus, and field preparation and alignment), in order for magnetic dipole moment (MDM) excitation to exceed direct charge excitation "background".

\section{Longitudinal polarimetry}

The possibility of resonant longitudinal polarimetry has been considered by numerous authors $[1,2,3,4,5]$.

Our proposed apparatus is illustrated by the photographs of a single cell, and a 4-cell longitudinal polarimeter, shown in Figure 2. A basic resonator cell is a several centimeter long copper split-cylinder, with gap serving as the capacitance $C$ of, for example, a $1.75 \mathrm{GHz} L C$ oscillator, with inductance $L$ provided by the conducting cylinder acting as a single turn solenoid. The photos show split-ring resonators built and tested at $\mathrm{UNM}$, resonant at $2.5 \mathrm{GHz}$, close to the design frequency. This resonator design (introduced by Hardy and Whitehead [6, 7] in 1981) and has been widely used for NMR measurements.
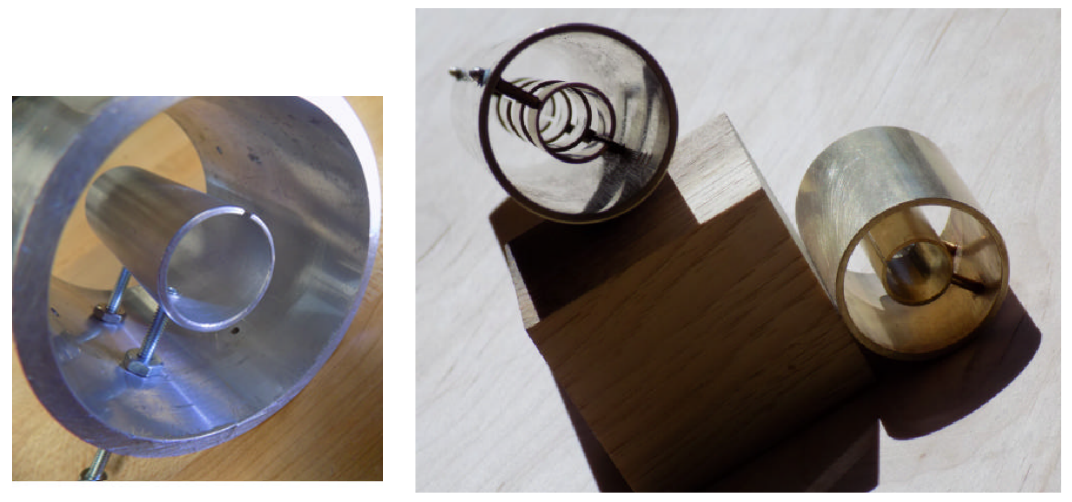

Figure 2: Photographs (with ends removed for visibility) of a single cell, on the left, along with a 4-cell longitudinal, split-cylinder polarimeter, on the right.

Figure 3 shows a longitudinally polarized electron beam (with spin polarizations represented schematically). A frequency $f_{0}$ train of longitudinally polarized bunchs of electrons in a linac beam passes through the split-cylinder resonator. The split cylinder can be regarded as a one turn solenoid. The bunch polarization toggles, bunch-to-bunch, between directly forward and directly 
backward. The resonator harmonic number relative to $f_{0}$ is an odd number in the range from 1 to 11 . (Actually 11 has been adopted.) This beam preparation immunizes the resonator from direct charge excitation. Irrespective of polarization, the $\mathrm{A}+\mathrm{B}$-combined bunch-charge frequencies will consist only of harmonics of $2 f_{0}=0.5 \mathrm{GHz}$, capable, at most, of only weakly exciting the resonator(s).

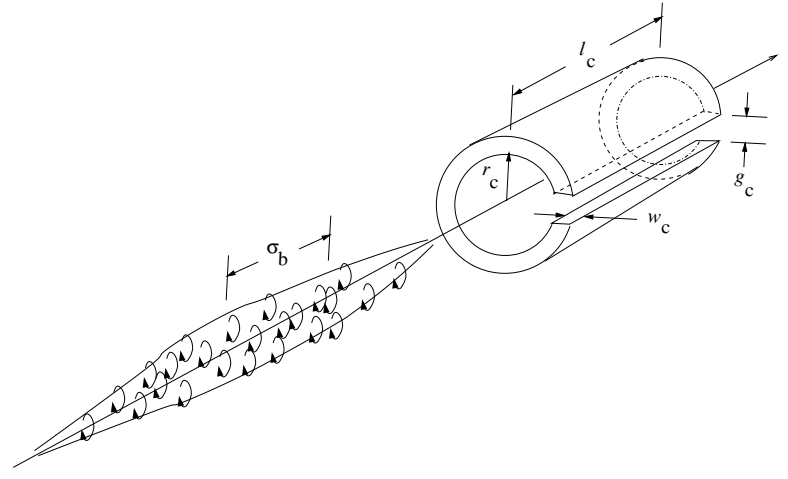

Figure 3: Perspective view of polarized beam bunch passing through the polarimeter. Dimensions are shown for the polarized proton bunch and the split-cylinder copper resonator. For the proposed test, using a polarized electron beam at Jefferson Lab, the bunch will actually be substantially shorter than the cylinder length, and have a beer can shape.

Figure 4 shows four such cells, regularly arrayed along the beam, to form a half-meter-long polarimeter. The magnetization of longitudinally-polarized electron bunches passing through the resonators coherently excites their fundamental oscillation mode and the coherently-summed "foreground" response from all resonators measures the polarization. "Background" due to direct charge excitation has been suppressed by arranging successive beam bunches to have alternating polarizations. This has moved the beam polarization frequency away from the direct beam charge frequency. Charge-insensitive resonator design, modulation-induced sideband excitation, and synchronous detection, permit the magnetization foreground to be isolated from spurious, chargeinduced background.

Figure 5 shows the signal processing electronics. The timing and synchronism of multiple bunches passing throught the polarimeter is illustrated by a space time diagram in Figure 6 . The resonator frequency is matched to the bunch velocity such that bunch entrance and exit impulses add constructively.

\subsection{Resonator parameters}

Treated as an $L C$ circuit, the split cylinder inductance is $L_{c}$ and the gap capacity is $C_{c}$. The highly conductive split-cylinder can be treated as a one-turn solenoid. For symplicity, minor corrections due to the return flux reluctance are not included in formulas given here. In terms of its current $I$, the magnetic field $B$ is given by

$$
B=\mu_{0} \frac{I}{l_{c}}
$$

The magnetic energy $W_{m}$ can be expressed in terms of $B$ or $I$;

$$
W_{m}=\frac{1}{2} \frac{B^{2}}{\mu_{0}} \pi r_{c}^{2} l_{c}=\frac{1}{2} L_{c} I^{2} .
$$



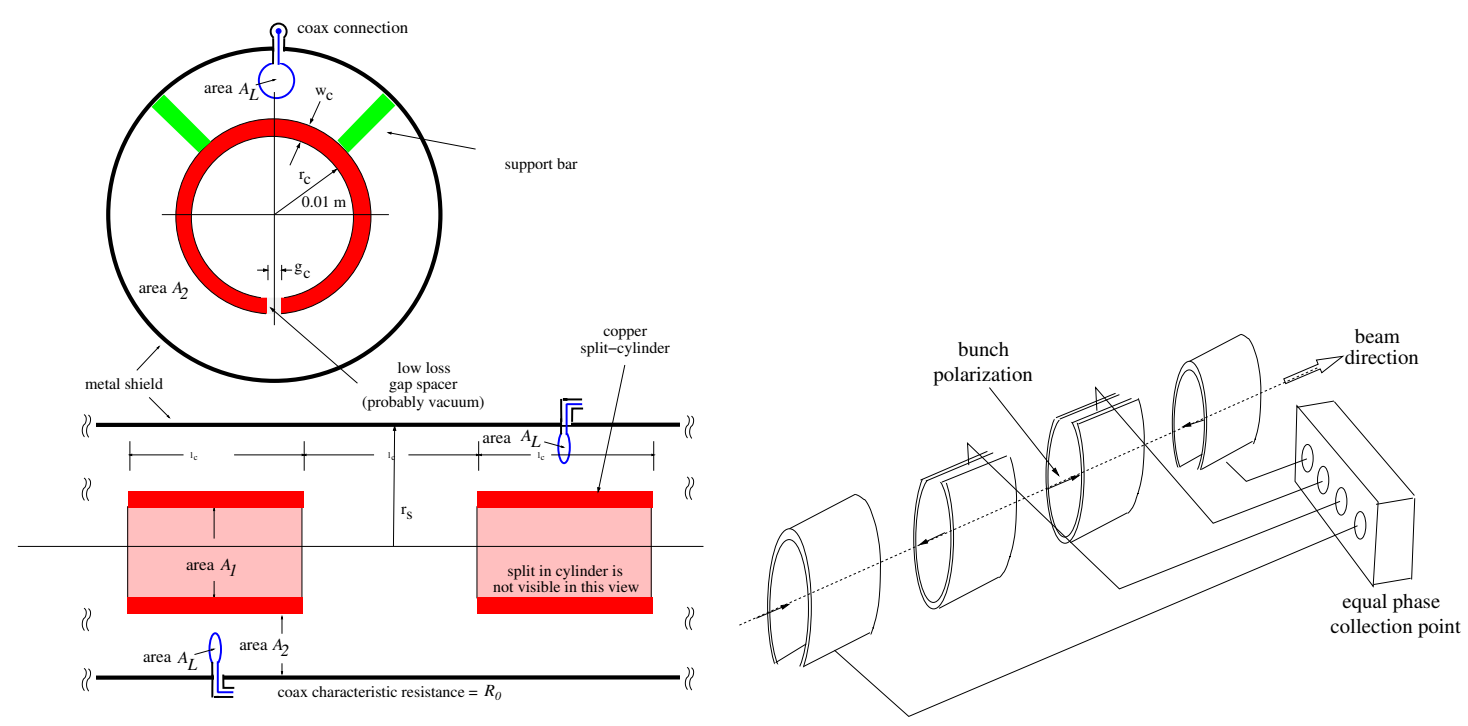

Figure 4: On the left are end and side views of two resonant split-cylinder polarimeter cells. Signals from individual resonators are loop-coupled out to coaxial cables and, after matched delay, added, as shown on the right. Cable lengths are arranged so that beam polarization signals add constructively.

The self-inductance is therefore

$$
L_{c}=\mu_{0} \frac{\pi r_{c}^{2}}{l_{c}}
$$

The gap capacitance (with gap $g_{c}$ reckoned for vacuum dielectric and fringing neglected) is

$$
C_{c}=\varepsilon_{0} \frac{w_{c} l_{c}}{g_{c}}
$$

Because the numerical value of $C_{c}$ will be small, this formula is especially unreliable as regards its separate dependence on $w_{c}$ and $g_{c}$. Furthermore, for low frequencies the gap would contain dielectric other than vacuum. Other resonator parameters, with proposed values, are given in Table 1.

\subsection{Local Lenz law (LLL) approximation}

A local Lenz law approximation for calculating the current induced in split cylinder by an electron bunch entering a split-cylinder resonator, treated as a one turn solenoid, is illustrated in Figure 7. The electron bunch is assumed to have a beer can shape, with length $l_{b}$ and radius $r_{b}$. Lenz's law is applied to the local overlap region of length $\Delta z$. Flux due to the induced Lenz law current exactly cancels the flux due to the Ampère bunch polarization current.

The magnetization $\mathbf{M}$ within length $\Delta z$ of a beam bunch (due to all electron spins in the bunch pointing, say, forward) is ascribed to azimuthal Amperian current $\Delta I_{b}=i_{b} \Delta z$. The bunch transit time is shorter than the oscillation period of the split cylinder and the presence of the gap in the cylinder at first produces little suppression of the Lenz's law current. $\Delta I_{L L}=i_{L L} \Delta z$ is the induced azimuthal current shown in the (inner skin depth) of the cylinder. To prevent any net flux from being present locally within the section of length $\Delta z$, the flux due to the induced Lenz law current must cancel the Ampère flux. 


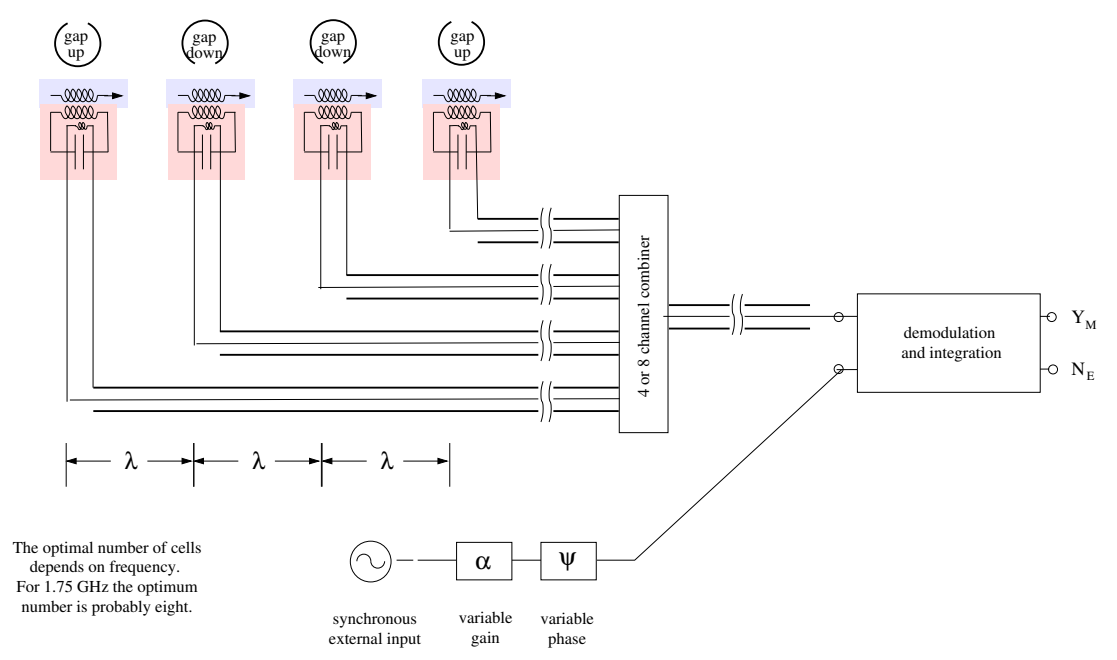

Figure 5: Circuit diagram for a circuit that coherently sums the signal amplitudes from four (or eight) polarimeter cells. Excitation by passing beam bunches is represented by inductive coupling. Quadrature signal separation routes in-phase signals to the $Y_{E}$ ("Yes it is magnetic-induced") output, and out-of-phase, quadrature signals to the $N_{E}$ ("No it is not magnetic-induced") output. The external coherent signal processing functionality to achieve this separation is indicated schematically by the box labelled "demodulation and integration".

Lenz law current per longitudinal length $i_{L L}$ induces Lenz law magnetic field $B_{L L}=\mu_{0} i_{L L}$ causing magnet flux through the cylinder

$$
\phi_{L L}=\mu_{0} \pi r_{c}^{2} i_{L L}
$$

Jackson [8] says the magnetic field $\mathbf{B}_{\mathbf{b}}$ within the polarized beam bunch is equal to $\mu_{0} \mathbf{M}_{\mathbf{b}}$ which is the magnetization (magnetic moment per unit volume) due to the polarized electrons.

$$
B_{b}=\mu_{0} M_{B}=\mu_{0} \frac{N_{e} \mu_{B}}{\pi r_{b}^{2} l_{b}},
$$

where $N_{e}$ is the total number of electrons in each bunch. The flux through ring thickness $\Delta z$ of this segment of the beam bunch is therefore

$$
\phi_{b}=B_{b} \pi r_{b}^{2}=\mu_{0} \frac{N_{e} \mu_{B}}{l_{b}} .
$$

Since the Lenz law and bunch fluxes have to cancel we obtain

$$
i_{L L}=-\frac{N_{e} \mu_{B}}{l_{b}} \frac{1}{\pi r_{c}^{2}}
$$

For a bunch that is longitudinally uniform (as we are assuming) we can simply take $\Delta z$ equal to bunch length $l_{b}$ to obtain

$$
I_{L L}=i_{L L} l_{b}=-\frac{N_{e} \mu_{B}}{\pi r_{c}^{2}}
$$

With the bunch fully within the cylinder, $I_{L L}$ "saturates" at this value. 


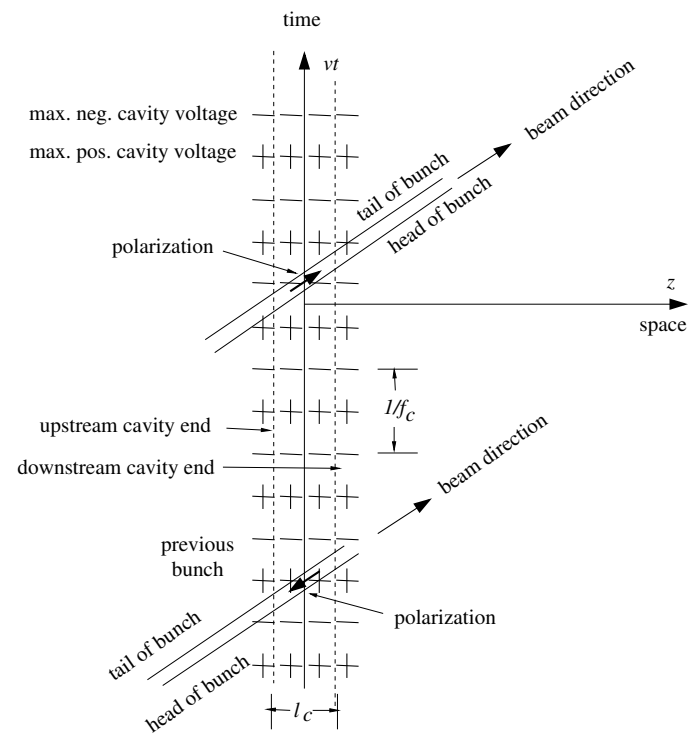

Figure 6: Space-time plot showing entry by the front, followed by exit from the back of one bunch, followed by the entrance and exit of the following bunch. Bunch separations and cavity length are arranged so that cavity excitations from all four beam magnetization exitations are perfectly constructive. The rows ++++ and - - - represent equal time contours of maximum or minimum $V_{C}, E_{\phi}, d B_{z} / d t$, or $d I_{C} / d t$, all of which are in phase

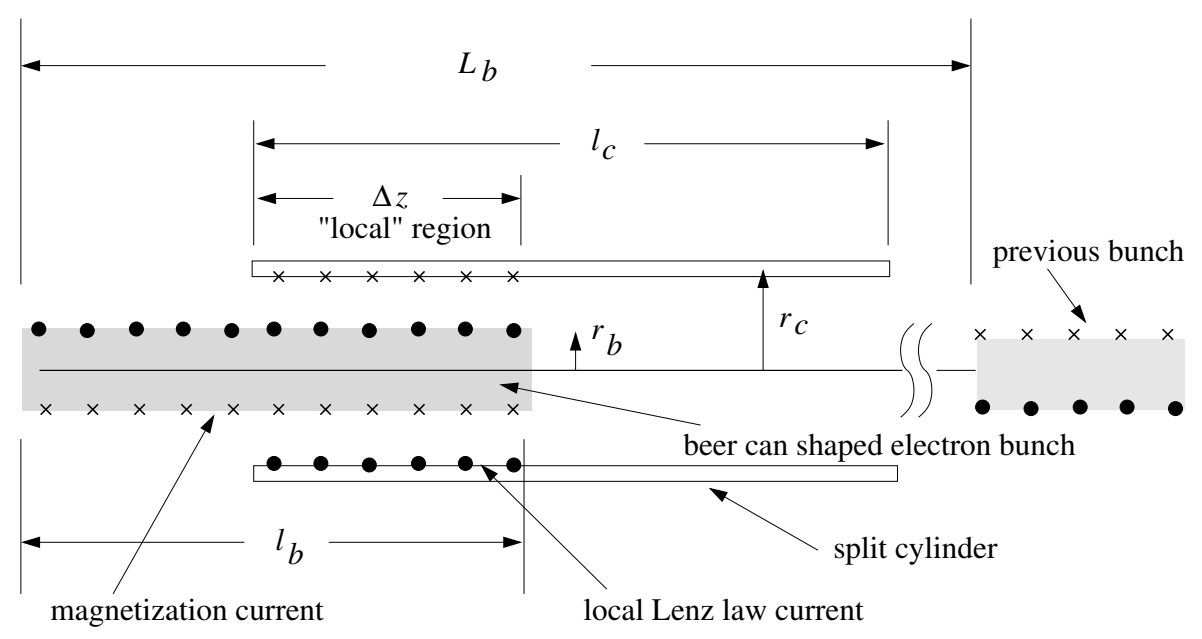

Figure 7: Figure illustrating the application of Lenz's Law to obtain the voltage induced in a split-cylinder resonator, by the passage of a longitudinally polarized bunch.

The bunch is short (i.e. $l_{b}<<l_{c}$ ) so the linear build up of $I_{L L}$ can be ascribed to a constant applied voltage $V_{L L}$ required to satisfy Faraday's law. For a CEBAF $I_{e}=160 \mu \mathrm{A}, 0.5 \mathrm{GHz}$ bunch frequency beam the number of electrons per bunch is approximately $2 \times 10^{6}$ and the Lenz law current is

$$
I_{L L}^{\max }=-\frac{N_{e} \mu_{B}}{\pi r_{c}^{2}} \quad\left(\stackrel{\text { e.g. }}{=}-5.9078 \times 10^{-14} \mathrm{~A}\right) .
$$

The same excess charge is induced on the capacitor during the bunch exit from the cylinder at which time the resonator phase has reversed. The total excess charge that has flowed onto the capacitor 


\begin{tabular}{|c|c|c|c|c|}
\hline $\begin{array}{c}\text { parameter } \\
\text { name }\end{array}$ & $\begin{array}{c}\text { parameter } \\
\text { symbol }\end{array}$ & formula & unit & value \\
\hline cylinder length & $l_{c}$ & & $\mathrm{~m}$ & 0.04733 \\
cylinder radius & $r_{c}$ & & $\mathrm{~m}$ & 0.01 \\
gap height & $g_{c}$ & & $\mathrm{~m}$ & 0.00103943 \\
wall thickness & $w_{c}$ & & $\mathrm{~m}$ & 0.002 \\
\hline capacitance & $C_{c}$ & $\varepsilon_{0} \frac{w_{c} l_{c}}{g_{c} / \varepsilon_{r}}$ & $\mathrm{pF}$ & 0.47896 \\
inductance & $L_{c}$ & $\mu_{0} \frac{\pi r_{c}^{2}}{l_{c}}$ & $\mathrm{nH}$ & 7.0213 \\
resonant freq. & $f_{c}$ & $1 /\left(2 \pi \sqrt{L_{c} C_{c}}\right)$ & $\mathrm{GHz}$ & 2.7445 \\
resonator wavelength & $\lambda_{c}$ & $c / f_{c}$ & $\mathrm{~m}$ & 0.10923 \\
\hline copper resistivity & $\rho_{\mathrm{Cu}}$ & & $\mathrm{ohm}-\mathrm{m}$ & $1.68 \mathrm{e}-8$ \\
skin depth & $\delta_{s}$ & $\sqrt{\rho_{\mathrm{Cu}} /\left(\pi f_{c} \mu_{0}\right)}$ & $\mu \mathrm{m}$ & 1.2452 \\
eff. resist. & $R_{c}$ & $2 \pi r_{c} \rho_{\mathrm{Cu}} /\left(\delta_{s} l_{c}\right)$ & $\mathrm{ohm}$ & 0.017911 \\
unloaded. qual. factor & $\mathscr{Q}$ & & & 6760.0 \\
\hline effective qual. fact. & $\mathscr{Q} / h_{c}$ & & & 643.65 \\
bunch frequency & $f_{A}=f_{B}=f_{0}$ & & $\mathrm{GHz}$ & 0.2495 \\
cavity harm. number & $h_{c}$ & $f_{c} / f_{0}$ & & 11 \\
electron velocity & $v_{e}$ & $c \sqrt{1-(1 / 2)^{2}}$ & $\mathrm{~m} / \mathrm{s}$ & $2.5963 \mathrm{e} 8$ \\
cavity transit time & $\Delta t$ & $l_{c} / v_{e}$ & $\mathrm{~ns}$ & 0.18230 \\
transit cycle advance & $\Delta \phi_{c}$ & $f_{c} \Delta t$ & & 0.50032 \\
entry cycle advance & & $\Delta \phi_{c} l_{b} / l_{c}$ & & 0.15011 \\
\hline electrons per bunch & $N_{e}$ & & & $2.0013 \times 10^{6}$ \\
bunch length & $l_{b}$ & & $\mathrm{~m}$ & 0.0142 \\
bunch radius & $r_{b}$ & & $\mathrm{~m}$ & 0.002 \\
\hline
\end{tabular}

Table 1: Resonator and beam parameters. The capacity has been calculated using the parallel plate formula. The true capacity is somewhat greater, and the gap $g_{c}$ will have to be adjusted to tune the natural frequency. When the A and B beam bunches are symmetrically interleaved, the bunch repetition frequency (with polarization ignored) is $2 f_{0}$.

due to the bunch passage is then

$$
Q_{1}^{\max .} \approx I_{L L}^{\text {sat. }} \frac{l_{b}}{v_{e}} \quad\left(\stackrel{\text { e.g. }}{=}-3.2312 \times 10^{-24} \mathrm{C} .\right) .
$$

$Q_{1}^{\max }$ is the charge deposited on the resonator capacitance during a single bunch passage of a bunch with the nominal $\left(N_{e}=2 \times 10^{6}\right.$ electrons) charge.

If there were no further resonator excitations, the charge on the capacitor would oscillate between $-Q_{1}^{\text {max. }}$ and $+Q_{1}^{\text {max. }} . U_{1}^{\text {pol. }}$, the corresponding resonator energy, is the "foreground" quantity that (magnified by a resonant amplitude build-up factor $M_{r}^{2}$ ) provides the polarization measure in the form of steady-state energy $U^{\text {pol. }}$ stored on the capacitor;

$$
U^{\text {pol. }}=\frac{1}{2} \frac{Q_{1}^{\max .2}}{C_{c}} M_{r}^{2}=\left(M_{r}^{2} \times 1.0899 \times 10^{-35} \mathrm{~J}\right)
$$

\subsection{Lumped circuit analysis ot resonant build-up}

In a MAPLE program the excitation is modeled using "piecewise defined" trains of pulses shown in Figure 8. Bipolar pulses modeling entry to and exit from the resonator are obtained as the difference between two, time-displaced "top hat" pulse trains. Pulsed excitation voltage pulse 

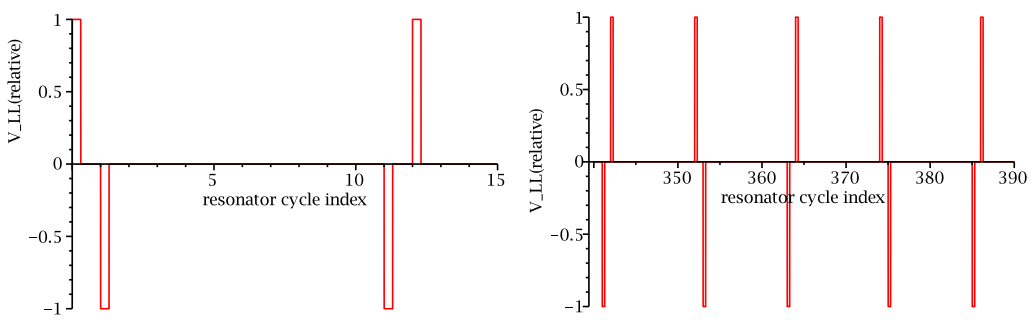

Figure 8: Early-time and later-time samples of the train of voltage pulses applied to the resonant cavity.

are caused by successive polarized bunch passages through the resonator. A few initial pulses are shown on the left, some later pulses are shown on the right. The units of the horizontal time scale are such that, during one unit along the horizontal time axis, the natural resonator oscillation phase advances by $\pi$. The second pulse starts exactly at 1 in these units. $h_{c}=11$ units of horizontal scale advance corresponds to a phase advance of $\pi$ at the $f_{A}=f_{B}=f_{0}=0.2495 \mathrm{GHz}$ "same-polarization repetition frequency".

Lumped constant representation [9] of the split-cylinder resonator as a parallel resonant circuit is shown in Figure 9. Voltage division in this series resonant circuit produces capacitor voltage

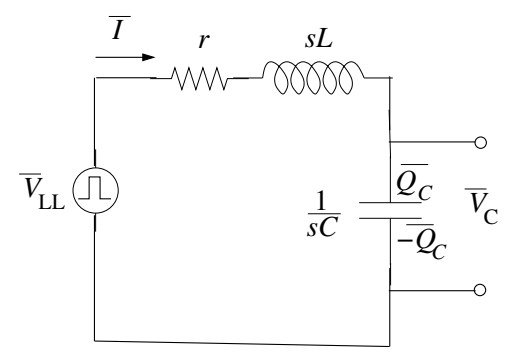

Figure 9: Equivalent circuit model for the resonant cavity.

transform $\bar{V}_{C}(s)$;

$$
\bar{V}_{C}(s)=\frac{1 /(C s)}{1 /(C s)+r+L s} \bar{V}_{L L}(s)=\frac{\bar{V}_{L L}(s)}{1+r s+C L s^{2}} .
$$

Synchronism of the input pulse train and the resonator response is illustrated in Figure 10. This comparison shows that the response is very nearly in phase with the excitation. Accumulation over early times of resonator excitation is shown in Figure 11. Accumulation over long times of resonator excitation is shown in Figure 12.

\subsection{Choice of resonator harmonic number and background rejection}

Resonator parameters for all possible odd-harmionics of the fundamental beam polarization frequency are shown in Table 2. We judge the highest harmonic, $h_{c}=11$, to be optimal. The expected saturation level resonator voltage is

$$
V_{C}^{\text {rcvr. }}=\frac{N_{\text {cell }}\left(\mathscr{Q} / h_{c}\right) Q_{1}^{\text {sat. }}}{C_{c}}=4.34 \times 10^{-9} \mathrm{~V}
$$

Accumulated over $100 \mathrm{~s}$, this is expected to be $31 \mathrm{db}$ above the thermal noise floor in a room temperature copper cavity. 


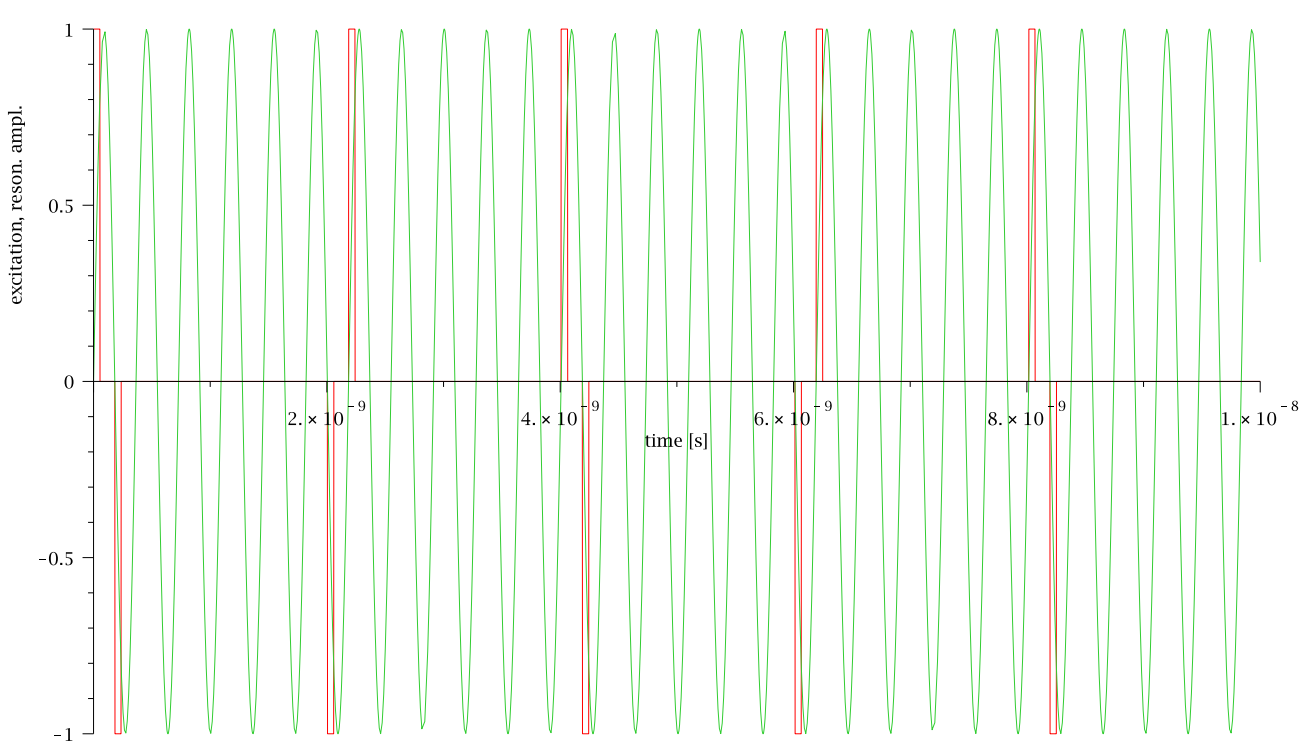

Figure 10: Alternating polarization excitation pulses superimposed on resonator response amplitude and plotted against time. Bunch separations are $2 \mathrm{~ns}$, bunch sepraration between same polarization pulses is $4 \mathrm{~ns}$. The vertical scale can represent $V_{C}, E_{\phi}, d B_{z} / d t$, or $d I_{C} / d t$, all of which are in phase.

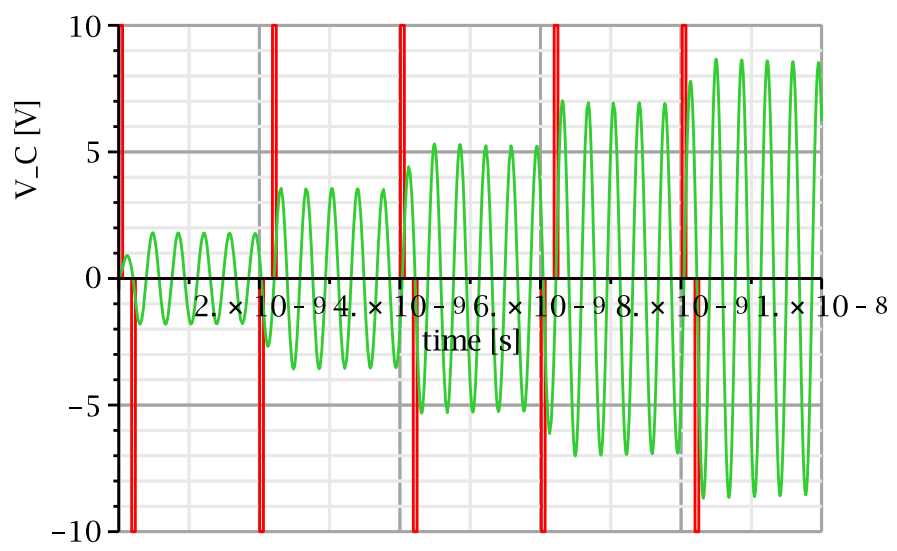

Figure 11: Accumulating capacitor voltage response $V_{C}$ while the first five linac bunches pass the resonator. The accumulation factor relative to a single passage, is plotted.

Estimated background rejection factors are shown in Table 3. According to these estimated factors the background direct excitation signal will be a few orders of magnitude below the foreground polarimetry signal. 


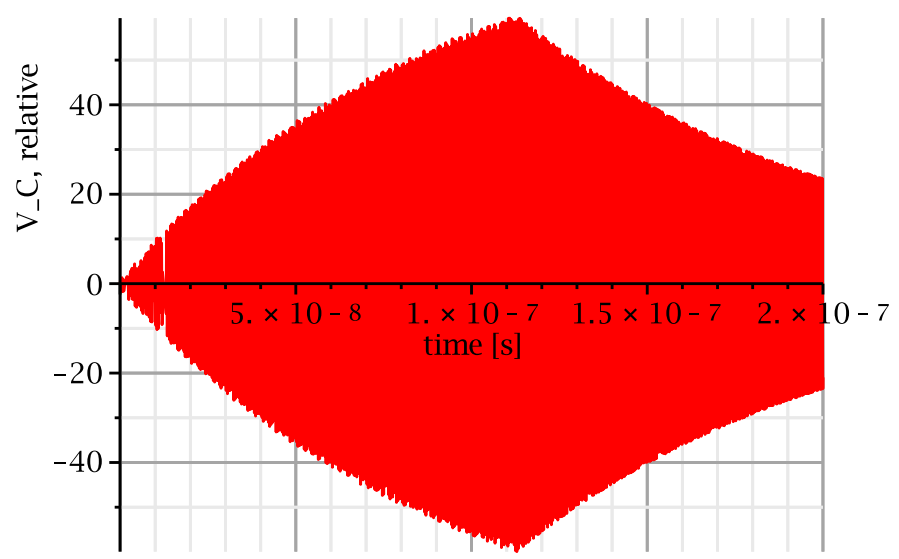

Figure 12: Relative resonator response to a train of beam pulse that terminates after about $110 \mathrm{~ns}$. After this time the resonator rings down at roughly the same rate as the build-up. The circuit parameters are those given in Table 1, except that the resistance for the plot is $r=10 r_{c}$. The true response build up would be greater by a factor of 10 , over a 10 times longer build-up time.

Table 2: Resonator parameters for all odd-harmionics of the fundamental beam polarization frequency.

\begin{tabular}{|c|c|c|c|c|c|c|c|}
\hline $\begin{array}{c}\text { parameter } \\
\text { harmonic numb. }\end{array}$ & $\begin{array}{c}\text { symbol } \\
h_{c}\end{array}$ & $\begin{array}{l}\text { unit } \\
\mathrm{GHz}\end{array}$ & 3 & 5 & 7 & 9 & 11 \\
\hline A,B bunch freq. & $f_{0}$ & $\mathrm{GHz}$ & 0.2495 & 0.2495 & 0.2495 & 0.2495 & 0.2495 \\
\hline resonant freq. & $f_{0}$ & $\mathrm{GHz}$ & 0.7485 & 1.2475 & 1.7465 & 2.2455 & 2.7445 \\
\hline dielectric & & & polyeth. & polyeth. & vacuum & vacuum & vacuum \\
\hline rel. diel. const. & $\varepsilon_{r}$ & & 2.30 & 2.30 & 1.00 & 1.00 & 1.00 \\
\hline numb. cells/m & $N_{\text {cell }}$ & $\approx / \mathrm{m}$ & 4 & 4 & 4 & 4 & 4 \\
\hline band width & $f_{c} / \mathscr{Q}$ & $\mathrm{kHz}$ & 286 & 277 & 309 & 351 & 388 \\
\hline quality factor & $\mathscr{Q}$ & & $2.61 e+03$ & $4.51 \mathrm{e}+03$ & $5.65 e+03$ & $6.40 \mathrm{e}+03$ & $7.08 \mathrm{e}+03$ \\
\hline effective qual. fact. & $M_{r}=\mathscr{Q} / h_{c}$ & & $8.72 \mathrm{e}+02$ & $9.01 \mathrm{e}+02$ & $8.07 e+02$ & $7.12 \mathrm{e}+02$ & $6.44 \mathrm{e}+02$ \\
\hline cyl. length & $l_{c}$ & $\mathrm{~cm}$ & 17.35 & 10.41 & 7.44 & 5.78 & 4.733 \\
\hline cyl. radius & $r_{c}$ & $\mathrm{~cm}$ & 1.0 & 1.0 & 1.0 & 1.0 & 1.000 \\
\hline gap height & $g_{c}$ & $\mathrm{~mm}$ & 1.305 & 2.021 & 0.709 & 1.171 & 1.750 \\
\hline wall thickness & $w_{c}$ & $\mathrm{~mm}$ & 10.0 & 5.0 & 2.0 & 2.0 & 2.0 \\
\hline capacitance & $C_{c}$ & $\mathrm{pF}$ & 27.076 & 5.245 & 1.859 & 0.874 & 0.479 \\
\hline inductance & $L_{c}$ & $\mathrm{nF}$ & 1670 & 3.10 & 4.47 & 5.74 & 7.02 \\
\hline skin depth & $\delta_{s}$ & $\mu m$ & 2.384 & 1.847 & 1.561 & 1.377 & 1.245 \\
\hline effective resistance & $R_{C}$ & $\mathrm{~m} \Omega$ & 2.55 & 5.49 & 9.09 & 13.26 & 17.91 \\
\hline cav. trans. time & $\Delta_{t}$ & ns & 0.668 & 0.401 & 0.286 & 0.223 & 0.182 \\
\hline entry cycle adv. & $\Delta_{t} f_{c} l_{b} / l_{c}$ & & 0.041 & 0.068 & 0.096 & 0.123 & 0.150 \\
\hline single pass energy & $U_{1, \max }$ & $\mathrm{J}$ & $1.9 \mathrm{e}-37$ & $1.0 \mathrm{e}-36$ & $2.8 \mathrm{e}-36$ & $6.0 \mathrm{e}-36$ & $1.1 \mathrm{e}-35$ \\
\hline sat. cap. volt. & $V_{C, \text { sat }}$ & V & $1.0 \mathrm{e}-10$ & $5.6 e-10$ & $1.4 \mathrm{e}-09$ & $2.6 \mathrm{e}-09$ & $4.3 e-09$ \\
\hline sat. cap. charge & $Q_{C, s a t}$ & $\mathrm{C}$ & $2.8 \mathrm{e}-21$ & $2.9 \mathrm{e}-21$ & $2.6 e-21$ & $2.3 e-21$ & $2.1 \mathrm{e}-21$ \\
\hline sat. ind. curr. & $I_{L, s a t}$ & A & $1.3 e-11$ & $2.3 e-11$ & $2.9 \mathrm{e}-11$ & $3.2 \mathrm{e}-11$ & $3.6 \mathrm{e}-11$ \\
\hline signal power & $P_{\text {sig }}$ & $\mathrm{W}$ & $4.39 \mathrm{e}-22$ & $4.03 \mathrm{e}-21$ & $1.28 \mathrm{e}-20$ & $2.72 \mathrm{e}-20$ & $5.0 \mathrm{e}-20$ \\
\hline therm. noise floor @ 1s & $P_{\text {noise }}$ & W & $4.05 \mathrm{e}-21$ & $4.05 \mathrm{e}-21$ & $4.05 \mathrm{e}-21$ & $4.05 \mathrm{e}-21$ & $4.05 e-21$ \\
\hline signal/noise at $1 \mathrm{~s}$ & $\log _{10}\left(P_{\text {sig }} / P_{\text {noise }}\right)$ & $\mathrm{db}$ & -9.65 & -0.01 & 4.99 & 8.27 & 10.88 \\
\hline signal/noise at $100 \mathrm{~s}$ & $"+20$ & $\mathrm{db}$ & 10.35 & 19.99 & 24.99 & 28.27 & 30.88 \\
\hline
\end{tabular}


Table 3: The first column gives set-up conditions which, if perfect, would completely suppress direct resonator excitation caused by the beam charge. The third and fourth columns give, respectively, installation specifications and the tuned-up improvement factors that are expected to be achievable operationally. The bottom line estimates the surviving background/foreground ratio.

\begin{tabular}{|c|c|c|c|c|}
\hline misalignment & $\begin{array}{c}\text { misalignment } \\
\text { factor } \\
\text { formula }\end{array}$ & $\begin{array}{c}\text { installation } \\
\text { specification }\end{array}$ & $\begin{array}{c}\text { operational } \\
\text { improvement } \\
\text { factor }\end{array}$ & $\begin{array}{c}\text { background } \\
\text { reduction } \\
\text { factor }\end{array}$ \\
\hline beam position & $\sqrt{\sigma_{x}^{2}+\sigma_{y}^{2}}$ & $<0.001 \mathrm{~m}$ & $/ 10^{2}$ & $1 \mathrm{e}-5$ \\
beam slope & $\sqrt{\sigma_{x^{\prime}}^{2}+\sigma_{y^{\prime}}^{2}}$ & $<0.001$ & $/ 10$ & $1 \mathrm{e}-4$ \\
A/B imbalance & $\Delta I_{\text {ave }} / I_{\text {ave }}$ & $<0.01$ & $/ 10$ & $1 \mathrm{e}-3$ \\
\hline pol. modulate & $\mathscr{S}^{\text {pol. }}$ & & $/ 10$ & $1 \mathrm{e}-1$ \\
slope modul & $\mathscr{S}^{\text {m.a. }}$ & & $/ 10$ & $1 \mathrm{e}-1$ \\
\hline noise/signal & $10^{10} \mathscr{S}^{\text {m.a. }} \mathscr{S}^{\text {pol. }} W_{1}^{\text {m.a. }} / U^{\text {pol. }}$ & & & $1 \mathrm{e}-4$ \\
\hline
\end{tabular}




\section{Transverse, Stern-Gerlach polarimetry}

A Jefferson Lab test is also proposed to detect Stern-Gerlach (SG) electron deflection, with a view toward non-destructive transverse polarimetry. As mentioned previously, the same alternating polarization beam preparation described earlier provides the main protection against direct resonator excitation by the beam charge. However, transverse polarimetry will depend on resonant BPM's completely unlike the resonators sensitive to longitudinal polarization.

Permanent magnet quadrupoles of the type to be used for the transverse polarimetry test beam line are shown in Figure 13. The polarimeter beam line, consisting, for example, of 8 small bore permanent magnet quadrupoles, (or half-quadrupoles) is shown in Figure 14. Parameter values

(a)

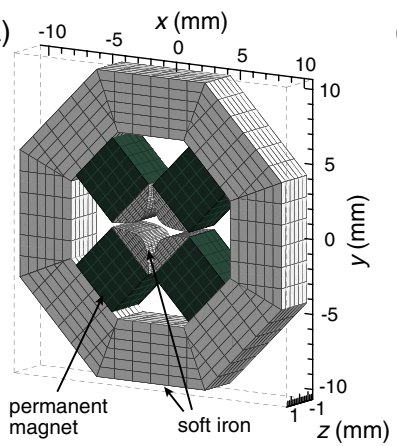

(b)

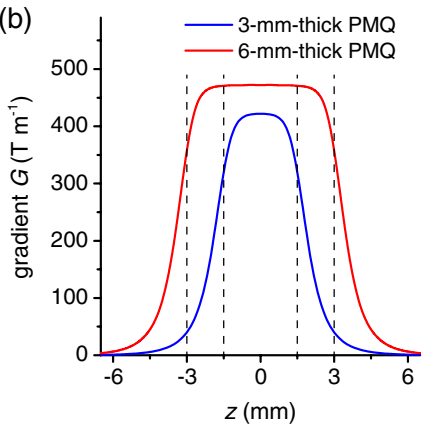

FIG. 5. (a) RADIA model of a 3-mm-thick PMQ magnet and (b) the calculated on-axis focusing gradient of a 3-mm and a 6-mm PMQ. Dashed lines indicate the physical boundaries of the 3- and 6-mm-thick PMQs.

Figure 13: Permanent magnet quadrupoles to be used for the transverse polarimetry test beam line. The included figure caption is copied from $\mathrm{Li}$ and Musumeci [10].

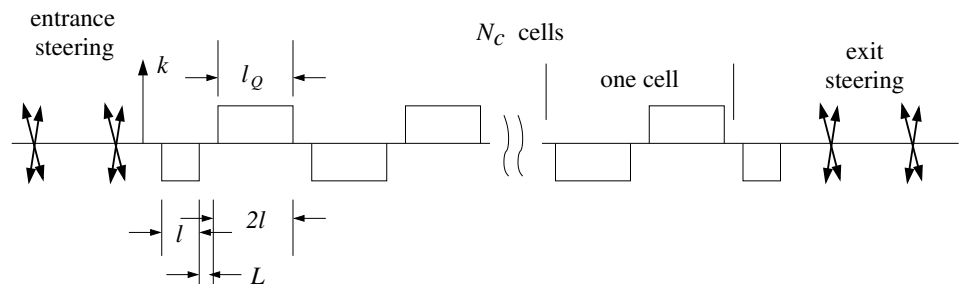

Figure 14: Focusing optics of the transverse polarimetry test beam line.

for numerical calculations in this paper include: quadrupole length $l_{Q}=2 l=0.02 \mathrm{~m}$; quadrupole separation $L=0.005 \mathrm{~m}$; and number of FODO cells $N_{c}=4$. Entrance and exit steering, indicated by crossed arrows, is needed to correct for quadrupole misalignment steering.

Positive Stern-Gerlach detection might seem to "refute" a famous Bohr-Pauli assertion that the Stern-Gerlach experiment cannot be performed with electrons. But not really! The quotation marks on "refute" acknowledge that Bohr and Pauli had no knowledge of modern technical capabilities. More important, the most essential aspect of their claim - that electrons cannot be "separated" by 
their spin state with an SG apparatus - is not disputed. Polarization-independent defocusing of the (finite-emittance) beam dwarfs any achievable separation into a spin-up and a spin-down beam.

It should, however, be possible to measure the polarization state of an electron beam by measuring the centroid deflection caused by its magnetization. This is what needs to be demonstrated. If and when this has been achieved, a high analysing power, non-destructive form of (transverse) polarimetry will have been demonstrated

For the initial test described in this paper we choose $N_{c}=4$. But, for an eventual apparatus, $N_{c}$ could be several times greater, depending on tolerance issues to be discussed. Since the design uses permanent magnets, any realization of the design is static, specific to a particular electron beam energy. But the design scales easly to other energies and parameter choices. The assumed quadrupoles are patterned after permanent magnet quadrupoles described in papers by Li, Musumeci, Maxson and others $[10,12]$.

During passage through a short quadrupole, the bend radius is determined by the centripetal force equation,

$$
\frac{p v}{r}=e v B=e v \frac{\partial B_{x}}{\partial x} x
$$

Re-arranging this equation, the integrated particle deflection angle during passage is

$$
\theta=\frac{l_{Q}}{r}=\frac{c l_{Q}\left\langle\partial B_{x} / \partial x\right\rangle x}{p c / e},
$$

For a quadrupole of strength (i.e. inverse focal length) $q=1 / f$, the deflection angle is $\pm q x$ where

$$
\begin{aligned}
q= \pm \frac{\theta}{x} & = \pm \mathscr{C}_{\gamma}\left(3 \times 10^{8}\right) /\left(0.511 \times 10^{6}\right)\left[\frac{l_{Q}\left\langle\partial B_{x} / \partial x\right\rangle}{\gamma_{e}}\right] \\
& \approx \pm 587 \mathrm{~T}^{-1} \mathrm{~m}^{-1}\left[\frac{l_{Q}\left\langle\partial B_{x} / \partial x\right\rangle}{\gamma_{e}}\right] .
\end{aligned}
$$

(Here $\mathscr{C}_{\gamma}$ is a number close to 1 , incapsulating various small effects that are being neglected, which is dropped from subsequent formulas.) The $\gamma_{e}$ factor inside the square bracket "cancels" the momentum dependence, allowing the lens strength to be expressed as an inverse focal length. (For fully relativistic electrons) the lens can be treated as purely geometric (i.e. independent of momentum) by varying $\partial B_{x} / \partial x$ proportional to $\gamma_{e}$. But this scaling fails when the magnetic field gradient cannot be increased further ! For this paper we take $l_{q}=0.02 \mathrm{~m}$ and (already achievable) field gradient $\partial B_{x} / \partial x=500 \mathrm{~T} / \mathrm{m}$ as nominal values. Higher field gradient, $\partial B_{x} / \partial x=1000 \mathrm{~T} / \mathrm{m}$, at shorter length, $l_{Q}=0.01 \mathrm{~m}$ is expected to be achievable [12]. This would yield the same length-strength product of $10 \mathrm{~T}$, but be more useful in the (important) sense of allowing a lens of the same strength to be shorter relative to its focal length.

Limited only by the maximum achievable permanent magnetic field gradient, even with careful element alignment and coherent multiplication of the displacement by the number of quadrupoles in the beam line, the Stern-Gerlach deflection can be expected to be only comparable in magnitude with deflection caused by misaligned quadrupoles. As already explained, this spurious excitation will be strongly suppressed by the interleaving of opposite-polarization A and B beams. This shifts the spectral frequency of the SG deflection to one half the spectral frequency of the spurious deflection, which will allow the SG contribution to be isolated in a frequency-sensitive BPM. 


\subsection{Beam line optics}

Optical properties of the proposed beam line are shown in the following figures. Figure 15 shows beta functions for the full beam line. Figure 16 shows the beta functions in the critical

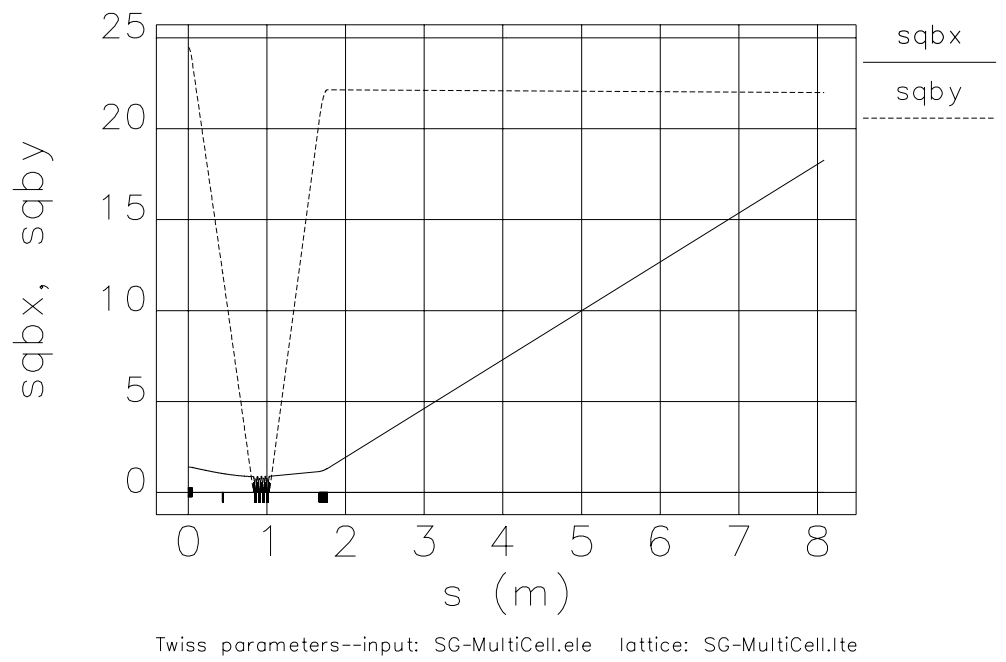

Figure 15: Beta functions for the Stern-Gerlach detection beam line. The length of the beam line is as long as possible consistent with the requirement that the rms beam size is conservatively smaller than the vacuum chamber radius. An SG-detecting BPM is located as far along the beam line as possible.

central section, which is the source region for the SG deflection. Only $N_{c}=4$ cells for the FODO section are shown, but $N_{c}$ could be increased with little effect on the matching. $N_{c}$ is limited, however, by the fact that the same optics that magnifies the SG deflection also magnifies the sensitivity to transverse beam displacement injection error.

The quadrupole at $s=1.72 \mathrm{~m}$ (at a distance $L_{\text {coll. }}=0.8 \mathrm{~m}$ from the center of the FODO lattice) is needed to restrict the growth of the defocussed transverse coordinate. But it also has the (minor) beneficial effect of magnifying the SG deflection. (See Eq. (3.8) below.) At low electron energy the beam emittance may limit the exit drift length to be shorter than shown in Figure 15, in order to prevent beam loss before the beam passes through the BPM's.

Figure 17 shows the accumulation of phase advances through the SG deflection source region. Since $25 / 8=3.125$, one sees that the phase advances per half cell are quite close to the value of 180 degrees, the maximum value that could be stable for arbitrarily large value of $N_{c}$. It is also the value for which all SG deflections superimpose constructively.

While discussing the energy dependence of the polarimetry one has to include the effect of adiabatic dampling, which causes the beam emittances to shrink proportional to $\gamma_{e}$. For fixed $q$, this produces a $\gamma_{e}^{1 / 2} \mathrm{SG}$ enhancement factor with increasing $\gamma_{e}$. The polarimetry capability for fixed beam line parameters "saturates" when the quadrupole strength required to produce the necessary focal length is no longer physically achievable.

Using the ELEGANT program, the focal lengths of the individual quadrupoles in the FODO line tuned for $\pi$ phase advance per half cell are

$$
q=k l_{Q}=68.1 \mathrm{~m}^{-1} .
$$




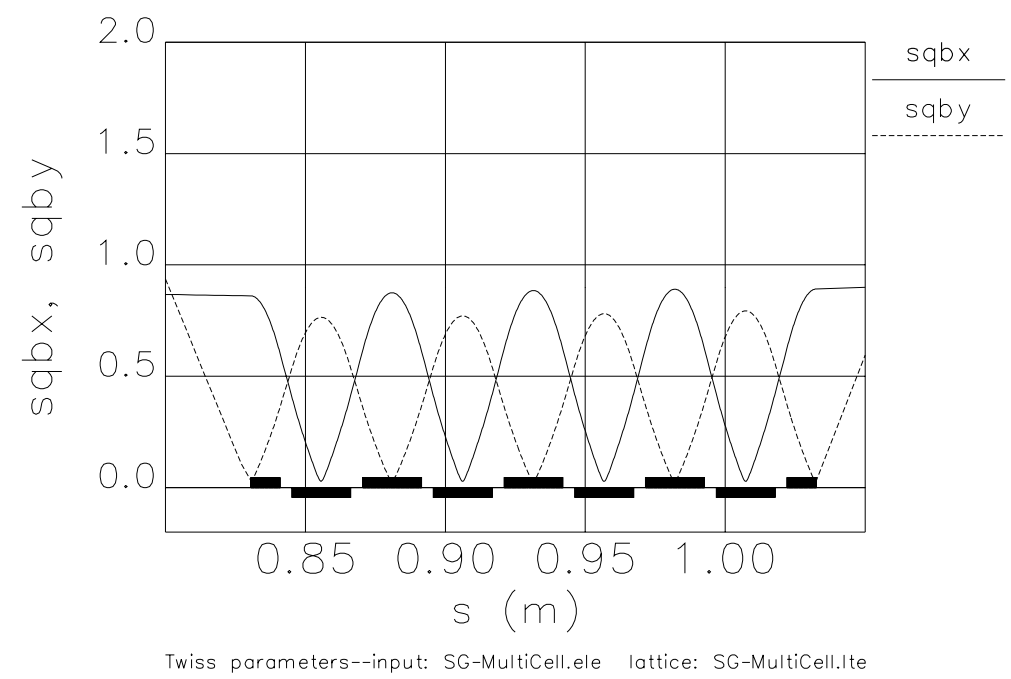

Figure 16: Optics in the central, periodic, multiple cell FODO section which is the coherent source of the SG deflections. The full quadrupole lengths are $l_{Q}=2 l=0.02 \mathrm{~m}$ and the quad separation distances are $L=0.005 \mathrm{~m}$. So the full cell length is $L_{\text {cell }}=0.05 \mathrm{~m}$. The entrance and exit half-quadrupoles simplify the lattice matching without otherwise influencing the performance.
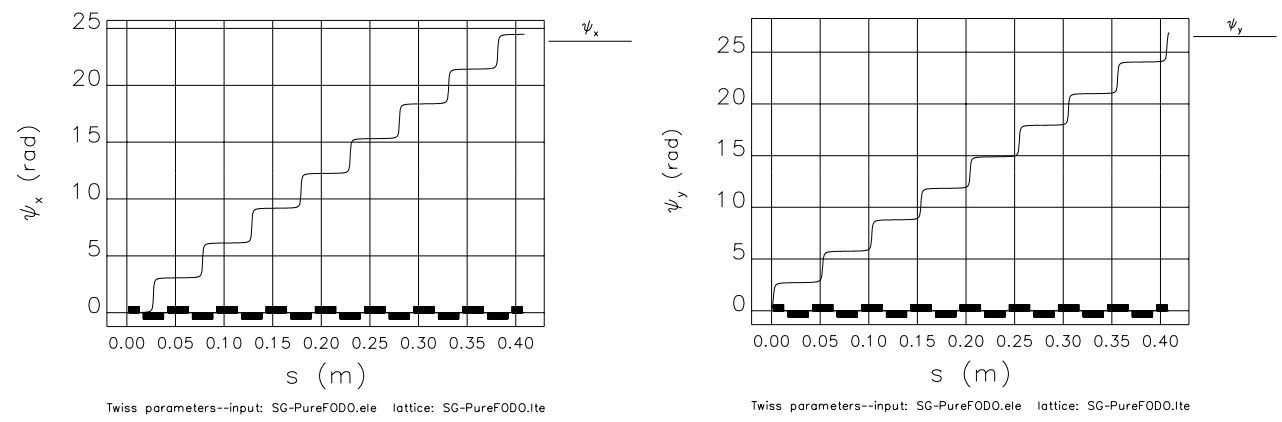

Figure 17: Phase advances $\psi_{x}$ and $\psi_{y}$ through a lattice with $N_{c}=8$ cells.

The corresponding focal length is $f=0.0147 \mathrm{~m}$-about 0.3 times the full cell length, as seems (intuitively) to be about right. Substitution of this $q$ value and $l_{Q}=0.02$ and rearranging produces

$$
l_{Q}\left\langle\partial B_{x} / \partial x\right\rangle=\frac{68.1 \mathrm{~m}^{-1}}{587 \mathrm{~T}^{-1} \mathrm{~m}^{-1}} \gamma_{e}, \quad \text { or } \quad\left\langle\frac{\partial B_{x}}{\partial x}\right\rangle=\frac{68.1 \mathrm{~m}^{-1}}{0.02 \mathrm{~m} \times 587 \mathrm{~T}^{-1} \mathrm{~m}^{-1}} \gamma_{e}=5.80 \mathrm{~T} / \mathrm{m} \gamma_{e} .
$$

If the practical limit for $\partial B_{x} / \partial x$ is $500 \mathrm{~T} / \mathrm{m}$, then the apparatus being described could act as a Stern-Gerlach polarimeter up to $\gamma_{e}=86$, or electron energy of $43 \mathrm{MeV}$.

\subsection{Stern-Gerlach orbit displacement}

The Stern-Gerlach deflection in a quadrupole is strictly proportional to the inverse focal lengths of the quadrupole;

$$
\Delta \theta_{x}^{S G}=\frac{\mu_{x}^{*}}{e c \beta} q_{x}, \quad \text { and } \quad \Delta \theta_{y}^{S G}=\frac{\mu_{y}^{*}}{e c \beta} q_{y} .
$$


The magnetic moments $\mu_{x}^{*}$ and $\mu_{y}^{*}$ differ from the Bohr magnetron $\mu_{B}$ only by $\sin \theta$ and $\cos \theta$ factors respectively. For a single quadrupole, the Stern-Gerlach-induced angular deflection is

$$
\Delta \theta^{S G}=\left(1.93 \times 10^{-13} \mathrm{~m}\right) q .
$$

To determine the downstream dispacement, one can use linear transfer matrix evolution;

$$
\left(\begin{array}{c}
\Delta x_{S G} \\
\cdot
\end{array}\right)=\left(\begin{array}{cc}
1 & L_{\mathrm{drift}} \\
0 & 1
\end{array}\right)\left(\begin{array}{cc}
1 & 0 \\
1.49 & 1
\end{array}\right)\left(\begin{array}{cc}
1 & L_{\mathrm{coll}} \\
0 & 1
\end{array}\right)\left(\begin{array}{c}
0 \\
\Delta \theta_{S G}
\end{array}\right),
$$

The collimating quadrupole strength is $1.49 / \mathrm{m}$. Completing the matrix multiplication yields

$$
\Delta x_{S G}=\left(0.8+2.19 L_{\mathrm{drift}}\right) \Delta \theta_{S G}
$$

The horizontal SG displacement is then given by

$$
\begin{aligned}
\Delta x_{\mathrm{SG}} & = \pm 2 N_{c}\left(1.93 \times 10^{-13} \mathrm{~m}\right) \times 68.1 \mathrm{~m}^{-1}\left(0.8 \mathrm{~m}+2.19 L_{\mathrm{drift}}\right) \\
& =1.59 \times 10^{-9} \mathrm{~m} .
\end{aligned}
$$

The \pm factor doubles the $S G$ displacement to $3.2 \mathrm{~nm}$; because the BPM is tuned to half the bunch passage frequency, it responds constructively to the oppositely polarized A and B beam bunches.

\subsection{Energy dependence of transverse polarimetry}

Expressing the quadrupole strength as an inverse focal length, as we have done, has had the effect of making the SG deflection independent of $\gamma$. Transverse beam size adiabatic damping enhances the energy dependence by a factor $\sqrt{\gamma}$. Even with the magnetic field gradient limited, the SG quadrupole lengths can be increased to preserve the optics described in this note, though with a longer FODO section. So the actual scaling with energy is such that the maximum achievable SternGerlach deflection increases as $\sqrt{\gamma}$ until the magnetic field gradient can no longer be increased. From there on, the response falls as $1 / \sqrt{\gamma}$ as the electron energy is increased.

As for the test at CEBAF, the most convenient energy remains to be determined. Discussions so far have assumed $500 \mathrm{KeV}$ electron kinetic energy, but this is for reasons of economy and accessibility, not because the SG signal is strongest at low energy. For the geometric parameters assumed in this note, the magnetic field gradient for $\gamma_{e}=2$ would be $12 \mathrm{~T} / \mathrm{m}$, far less than the maximum possible.

\subsection{Signal levels and noise suppression}

The resonant BPM relies on precise, on-axis, alignment of a cavity tuned to have an antisymmetric mode at the bunch charge passage frequency. Extreme selectivity is needed to separate the beam polarization signal from the spurious direct beam charge signal (in conjunction with misaligned equipment). Also the signal power induced in the position-sensitive cavity by SGinduced displacement has to exceed the inherent thermal noise "floor". This noise floor could, if necessary, be lowered by using cryogenic apparatus, but our estimates indicate that such an extreme measure is unnecessary. 
Performance of similar position-sensitive apparatus can be mentioned briefly. Pusch et al. report BPM measurement at the $0.1 \mathrm{~mm}$ level for beam currents greater than $250 \mathrm{pA}$. The proposed $\mathrm{J}$-Lab current is a million times greater. The off-axis shunt impedance of a resonant cavity is proportional to the square of the (beam-current $\times$ beam-displacement) product. By this estimate, the resonator excitation of $1 \AA$ will be at the noise floor. The SG displacement predicted for our beam line is approximately $30 \AA$.

International Linear Collider (ILC) motivated BPM performance design studies have shown that the $\pm 20 \AA$ beam position pulse-to-pulse reproduceability planned for effective ILC operation will be achievable. A CEBAF beam is $\mathrm{CW}$, with average current about five orders of magnitude higher than for the ILC BPM test at the KEK, ATF Test Facility. Averaging over longer times can reduce some noise sources. For these, the increased average beam current can improve the signal to noise by the square root of the current ratio. Also the ILC cavity discharging time is far shorter than the ATF repetition period, which makes it necessary for ILC prototyping to treat their BPM resonant response on a pulse-by-pulse basis. Our high bunch frequency permits phase-sensitive CW signal treatment.

\section{References}

[1] Ya. S. Derbenev, RF-resonance beam polarimeter, Part I. Fundamental concepts, Nuclear Instruments and Methods m Physics Research A 336, 12-15, 1993

[2] M. Conte, et al., The Stern-Gerlach interaction between a traveling particle and a time varying magnetic field, arXiv:physics/0003069v1 [physics.acc-ph], 2000

[3] P. Cameron, et al., An RF Resonance Polarimeter Phase I Proof of Principle Experiment, RHIC/AP/126

[4] C. Tschalaer, The Relativistic Stern-Gerlach Force, arXiv/papers/0802/0802:0154.pdf, 2008

[5] C. Tschalaer, Lorentz Transform of an Arbitrary Force Field on a Particle in its Rest Frame using the Hamilton-Lagrangian Formalism, BIR\#15-01, Bates Lab Report, 2015

[6] W. Hardy and L. Whitehead, Split-ring resonator for use in magnetic resonance from $200-2000 \mathrm{MHz}$, Review of Scientific Instruments, 52 (2) 213, 1981

[7] B. R. Johnson, Nuclear Spin Waves in Spin-Polarized Hydrogen, Cornell Ph.D. thesis, 1984

[8] J. Jackson, Classical Electrodynamics, 3rd edition, John Wiley, 1998

[9] C. Montgomery, R. Dicke, and E. Purcell, Principles of Microwave Circuits, McGraw-Hill, 1948

[10] R. Li and P. Musumeci, Single-Shot MeV Transmission Electron Microscopy with Picosecond Temporal Resolution, Physical Review Applied 2, 024003, 2014.

[11] D. Cesar et al., Demonstration of Single-Shot Picosecond Time-Resolved MeV Electron Imaging Using a Compact Permanent Magnet Quadrupole Based Lens, Phys. Rev. Lett. 17, 024801, 2016

[12] Private communication from P. Musumeci. 